\title{
Factors associated with improved survival following surgery for renal cell carcinoma spinal metastases
}

\author{
Rory J. Petteys, MD,, Steven M. Spitz, MD, ${ }^{1}$ C. Rory Goodwin, MD, PhD, ${ }^{2}$ Nancy Abu-Bonsrah, BS, ${ }^{2}$ \\ Ali Bydon, MD, ${ }^{2}$ Timothy F. Witham, MD, ${ }^{2}$ Jean-Paul Wolinsky, MD, ${ }^{2}$ Ziya L. Gokaslan, MD, ${ }^{3}$ and \\ Daniel M. Sciubba, MD²
}

\begin{abstract}
'Department of Neurosurgery, Georgetown University Hospital, Washington, DC; ${ }^{2}$ Department of Neurosurgery, The Johns Hopkins University, Baltimore, Maryland; and 'Department of Neurosurgery, Brown University School of Medicine, Providence, Rhode Island
\end{abstract}

\begin{abstract}
OBJECTIVE Renal cell carcinoma (RCC) frequently metastasizes to the spine, causing pain or neurological dysfunction, and is often resistant to standard therapies. Spinal surgery is frequently required, but may result in high morbidity rates. The authors sought to identify prognostic factors and determine clinical outcomes in patients undergoing surgery for RCC spinal metastases.
\end{abstract}

METHODS The authors searched the records of patients who had undergone spinal surgery for metastatic disease at a single institution during a 12-year period and retrieved data for 30 patients with metastatic RCC. The records were retrospectively reviewed for data on preoperative conditions, treatment, and survival. Statistical analyses (i.e., Kaplan-Meier survival analysis and log-rank test in univariate analysis) were performed with $\mathrm{R}$ version 2.15.2.

RESULTS The 30 patients ( 23 men and 7 women with a mean age of 57.6 years [range $29-79$ years]) had in total 40 spinal surgeries for metastatic RCC. The indications for surgery included pain $(70 \%)$ and weakness $(30 \%)$. Fourteen patients (47\%) had a Spinal Instability Neoplastic Score (SINS) indicating indeterminate or impending instability, and 6 patients $(20 \%)$ had a SINS denoting instability. The median length of postoperative survival estimated with Kaplan-Meier analysis was 11.4 months. Younger age $(p=0.001)$ and disease control at the primary site $(p=0.005)$, were both significantly associated with improved survival. In contrast, visceral $(p=0.002)$ and osseous $(p=0.009)$ metastases, nonambulatory status $(p=0.001)$, and major comorbidities $(p=0.015)$ were all significantly associated with decreased survival. Postoperative Frankel grades were the same or had improved in $78 \%$ of patients. Major complications occurred in 9 patients, and there were 3 deaths $(10 \%)$ during the 30-day in-hospital period. Three en bloc resections were performed. CONCLUSIONS Resection and fixation may provide pain relief and neurological stabilization in patients with spinal metastases arising from RCC, but surgical morbidity rates remain high. Younger patients with solitary spinal metastases, good neurological function, and limited major comorbidities may have longer survival and may benefit from aggressive intervention.

http://thejns.org/doi/abs/10.3171/2016.5.FOCUS16145

KEY WORDS carcinoma; renal cell; metastatic; prognosis; spine; Spinal Instability Neoplastic Score; SINS

$\Lambda$ PPROXIMATELY 1.6 million Americans will have a diagnosis of cancer in 2013, an annual incidence that has remained relatively unchanged for decades. ${ }^{1}$ Owing to notable improvements in treatment regimens and diagnostic capabilities, cancer patients with advanced metastatic disease are surviving longer. One of the most frequent sites of metastasis is the spine, and these spinal metastases are a significant cause of high morbidity rates among cancer patients. About $10 \%$ of cancer patients will develop symptomatic spinal metastases, with $10 \%$ of these patients undergoing some form of surgical treatment. . $^{2,324,33,35}$ Of note, $75 \%$ of spinal metastases typically result from 1 of 5 malignancies: lung, breast, prostate, renal, or thyroid carcinoma. ${ }^{8}$

Renal cell carcinoma (RCC) is a relatively uncommon but aggressive neoplastic disease that frequently metastasizes to other visceral organs, to local structures, and to the skeletal system. Approximately 65,000 new cases of kidney cancers-93\% of which are RCC-were predicted to be diagnosed in the United States in 2013, with more

ABBREVIATIONS RCC = renal cell carcinoma; SINS = Spinal Instability Neoplastic Score; TES $=$ total en bloc spondylectomy.

SUBMITTED March 31, 2016. ACCEPTED May 27, 2016.

INCLUDE WHEN CITING DOI: 10.3171/2016.5.FOCUS16145. 
than 13,000 deaths from RCC. ${ }^{1}$ Because of RCC's indolent nature, many patients already have metastatic lesions, including spinal metastases, when they initially present. Although RCC makes up only $2.5 \%$ of all cancers in adults, it is the fourth most common metastatic lesion in the spine after breast, lung, and prostate cancers. ${ }^{16,21}$ When osseous metastases are present, RCC typically has an aggressive course, resulting in limited expected survival (i.e., of only 12-22 months). ${ }^{12,22}$ However, there are reports of long overall survival in the setting of isolated RCC spinal metastases particularly with improved medical therapy..$^{20,25,27,29,30}$

Like other neoplastic diseases, RCC usually spreads to the spine through hematogenous dissemination or local invasion. Once metastatic disease is present, patients may have debilitating back or extremity pain from compression of the spinal cord or nerve roots; these patients also often have local inflammation and mechanical instability in the spine. Neurological deficits may also develop because of tumor growth into the vertebral canal or retropulsion of bone fragments as a result of pathological fractures due to the lytic nature of these metastatic lesions. Focused radiotherapy and chemotherapy may effectively treat pain that arises from tumor growth or inflammation, but these therapies cannot address mechanical instability or spinal cord compression from bony elements. Therefore, surgery remains necessary for many patients with metastatic spine lesions.

Research and development in recent decades have led to tremendous technical advancements in the treatment for spinal column disorders. New techniques and technologies have expanded the arsenal of the spine surgeon in the management of many conditions, including metastatic lesions. Several studies have demonstrated the benefits of surgery combined with systemic and adjuvant local therapies in alleviating pain and maintaining neurological function. ${ }^{7,11,13,19}$ Furthermore, some patients with RCC can be expected to have long, disease-free survival if their systemic disease burden is low and if aggressive resection is used. ${ }^{27}$ Here, in an effort to identify prognostic factors for RCC survival, we present demographic and survival data on 30 patients who underwent 40 spinal operations for metastatic RCC at a single institution over a 12-year period.

\section{Methods}

\section{Patient Selection}

We conducted a retrospective review of the records of all patients who underwent spinal surgery for metastatic disease at our institution from January 2000 to December 2011. The review yielded data on 30 patients in whom histopathological analysis had confirmed the presence of metastatic RCC. These patients had undergone a total of 40 spinal operations for metastatic RCC. Electronic medical records for these patients were reviewed, and data on patient demographics, preoperative neurological condition, functional status, primary disease location, systemic disease burden, other treatments, and intraoperative and postoperative events were collected. Collections of patient medical record data were conducted in accordance with our university's institutional review board.

\section{Surgical Procedures}

Before surgery, all patients were examined with MRI of the spine to visualize the extent of tumor involvement in the vertebral column and spinal canal. A systemic search for visceral and other osseous metastases was performed and involved a multidisciplinary team. This survey included abdominal and renal sonographies, CT of the chest, abdomen, and pelvis, and, in some cases, nuclear scintigraphy or PET. Computed tomography or MRI of the brain was performed when clinically indicated.

Indications for surgery included severe back or extremity pain, neurological dysfunction, radiographic or clinical signs of spinal instability, and the need for tissue diagnosis in patients with spinal metastasis of unknown primary origin. The Spinal Instability Neoplastic Score (SINS) was calculated for each patient. The operative approach was determined by the primary operating surgeons and ranged from decompressive laminectomy to en bloc resection with negative margins via spondylectomy. Internal fixation and stabilization were performed in all but 1 patient. Patients who underwent an instrumented fusion in the absence of tumor-related spinal instability or of impending tumor-related instability underwent this fusion because of instability caused by the surgical approach. Preoperative arterial embolization was performed in 24 patients (80\%). All patients were transferred to the intensive care unit immediately postoperatively for at least 1 day.

\section{Statistical Analysis}

Kaplan-Meier estimates were calculated to determine median length of survival and the presence of any statistically significant factors affecting survival. Additional analyses were performed with the log-rank test for univariate analysis. Statistical analyses were performed with $\mathrm{R}$ version 2.15.2 ( $\mathrm{R}$ Foundation for Statistical Computing).

\section{Results}

\section{Patient Characteristics}

Thirty patients underwent a total of 40 spine operations for the treatment of RCC spinal metastases. There were 23 men (76.7\%) and 7 women (23.3\%), with a mean age of 57.6 years (range 29-79 years). The indications for surgery included intractable back pain or radiculopathy in 21 patients (70\%), neurological dysfunction in 9 patients $(30 \%)$, isolated metastasis in 21 patients $(70 \%)$, and treatment failure diagnosed on routine imaging radiographs in 1 patient (3\%). Preoperative treatments such as spinal radiotherapy combined with systemic chemotherapy were performed in 15 patients $(50 \%)$ and immunotherapy in 12 patients (40\%). Preoperative variables and patient characteristics are shown in Table 1. Preoperative intraarterial particulate embolization was performed in 24 patients $(80 \%)$ before initial tumor resection. The preoperative treatments did not significantly affect survival.

\section{Overall Survival}

Data on overall survival are shown in Fig. 1. The median length of survival was 11.4 months after surgery (range 1 month to 9.9 years), and the 5-year survival rate, deter- 
TABLE 1. Demographic and background information*

\begin{tabular}{lcc}
\hline \multicolumn{1}{c}{ Variable } & Value & p Value† \\
\hline Mean age in yrs (range) & $57.6(29-79)$ & 0.001 \\
\hline Sex & & - \\
\hline Male & $23(76.7)$ & \\
\hline Female & $7(23.3)$ & \\
\hline Preop condition & & - \\
\hline Pain & $21(70.0)$ & 0.06 \\
\hline $\begin{array}{l}\text { Neurological dysfunction (Frankel } \\
\text { Grades A-D) }\end{array}$ & $9(30.0)$ & \\
\hline Ambulatory & $25(83.3)$ & 0.001 \\
\hline Primary site control & $19(63.3)$ & 0.005 \\
\hline Visceral metastases & $13(43.3)$ & 0.002 \\
\hline Extraspinal bony metastases & $7(23.3)$ & 0.009 \\
\hline Comorbidities & $3(10.0)$ & 0.015 \\
\hline Preop treatment & & \\
\hline Spine XRT & $15(50.0)$ & 0.96 \\
\hline Chemotherapy & $12(40.0)$ & 0.93 \\
\hline Embolization & $24(80.0)$ & - \\
\hline
\end{tabular}

$-=$ not determined $; \mathrm{XRT}=$ radiotherapy.

* Data represent number of patients (\%), unless indicated otherwise.

$\dagger p$ values $<0.05$ were considered statistically significant.

mined with the Kaplan-Meier method, was $10 \%$. Age at the time of surgery was a significant predictor of survival $(\mathrm{p}=0.001)$, with younger patients having longer survival. Local disease control, that is, nephrectomy with no evidence of residual disease at the primary site, was also significantly associated with increased survival $(p=0.005)$. In contrast, visceral $(\mathrm{p}=0.002)$ and other extraspinal osseous $(p=0.009)$ metastases were significant indicators for decreased survival, as were the inability to ambulate and the presence of neurological dysfunction (indicated by Frankel grades of A-D). Major preoperative comorbidities, including previous myocardial infarction, coronary artery bypass grafting, and concomitant malignancies (lymphoma and oropharyngeal squamous cell carcinoma), were present in 3 patients and were also associated with decreased survival $(p=0.015)$. None of the postoperative variables that were analyzed, including systemic chemotherapy, spinal radiotherapy, or local cancer recurrence, affected survival.

Most patients (70\%) in this series had back or radicular pain, and some patients (30\%) had neurological dysfunction (Table 1). Among the 9 patients with neurological impairment, in $7(78 \%)$ the condition had improved or was the same at the time of discharge from the hospital. The condition of 1 patient (11\%) was worse, and 1 patient $(11 \%)$ died from respiratory failure. Among the 21 patients with significant back or extremity pain, 17 patients $(81 \%)$ had less pain postoperatively as measured by self-reported use of analgesics and by visual analog pain scale assessments.

\section{Surgical Procedures}

The reported operative variables for each patient per-

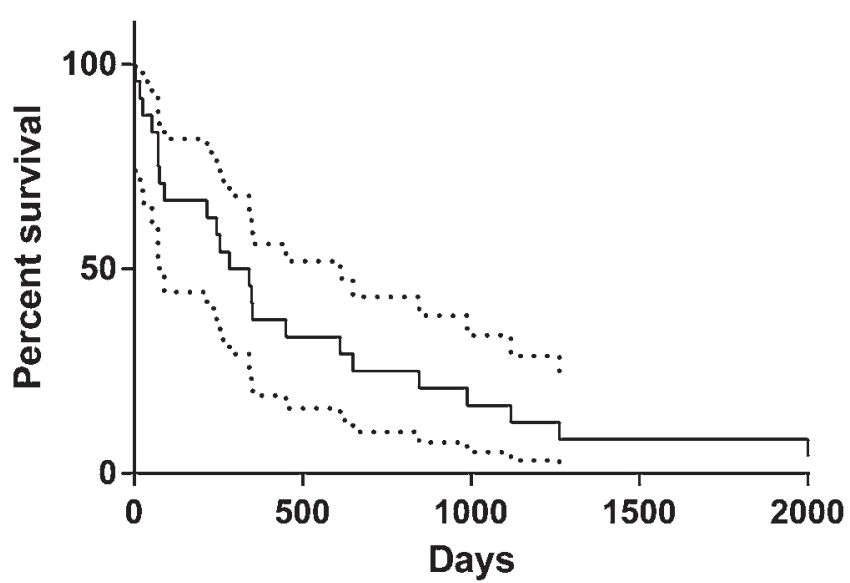

FIG. 1. Kaplan-Meier survival estimates for patients with RCC spinal metastases (dashed lines indicate $95 \% \mathrm{Cl}$ ).

tain to the initial surgery for spinal metastatic lesions, and subsequent surgeries were considered separately. The anatomical locations of the metastatic lesions were as follows: the cervical spine in 3 patients (10\%), thoracic spine in 18 $(60 \%)$, lumbar spine in $8(27 \%)$, and sacral spine in $1(3 \%)$. The surgical approach was determined by the operating surgeons on the basis of imaging findings, patient disease status, and associated comorbidities. Focal instability as determined by the presence of mechanical spine pain on presentation, movement on dynamic radiographs, or evidence of 2-column instability (i.e., kyphosis of $>20^{\circ}$ or vertebral body height loss $>50 \%$ ) were also indications for surgical intervention (Fig. 2). Patients having multiple consecutive levels of metastatic disease were also considered for a surgical intervention, as the spines in these patients have a higher propensity for focal instability. Instability was retrospectively verified with SINS criteria, and 14 patients $(47 \%)$ had a SINS denoting indeterminate or impending instability, and $6(20 \%)$ had a SINS denoting instability.

Internal fixation consisting of pedicle screws, rods, intervertebral cages, and anterior plates was performed as needed in all but 1 patient. An anterior approach alone was used in 6 patients (20\%), a posterior-only approach in 15 patients $(50 \%)$, and combined anteroposterior approach in 9 patients (30\%). Of the combined anteroposterior surgeries, $6(67 \%)$ were performed in a staged fashion. Circumferential decompression, through either a solely posterior or a combined anteroposterior approach, was performed in 21 patients (70\%). Three patients (10\%) underwent en bloc resection. The mean estimated blood loss was $3066 \pm 2785 \mathrm{ml}$, with an average of 8.3 blood or platelet transfusions over the course of each individual patient's hospital stay. The operative approach did not affect survival, although en bloc resection with negative margins did show a trend toward improved survival outcomes $(\mathrm{p}$ $=0.051$ ), likely reflecting selection bias because these patients had solitary metastases. However, 2 of these patients were alive at the time of this writing, and future analysis might show statistically significantly improved survival in patients treated with en bloc resection. The mean age of patients undergoing en bloc resection (54.3 years) did not 


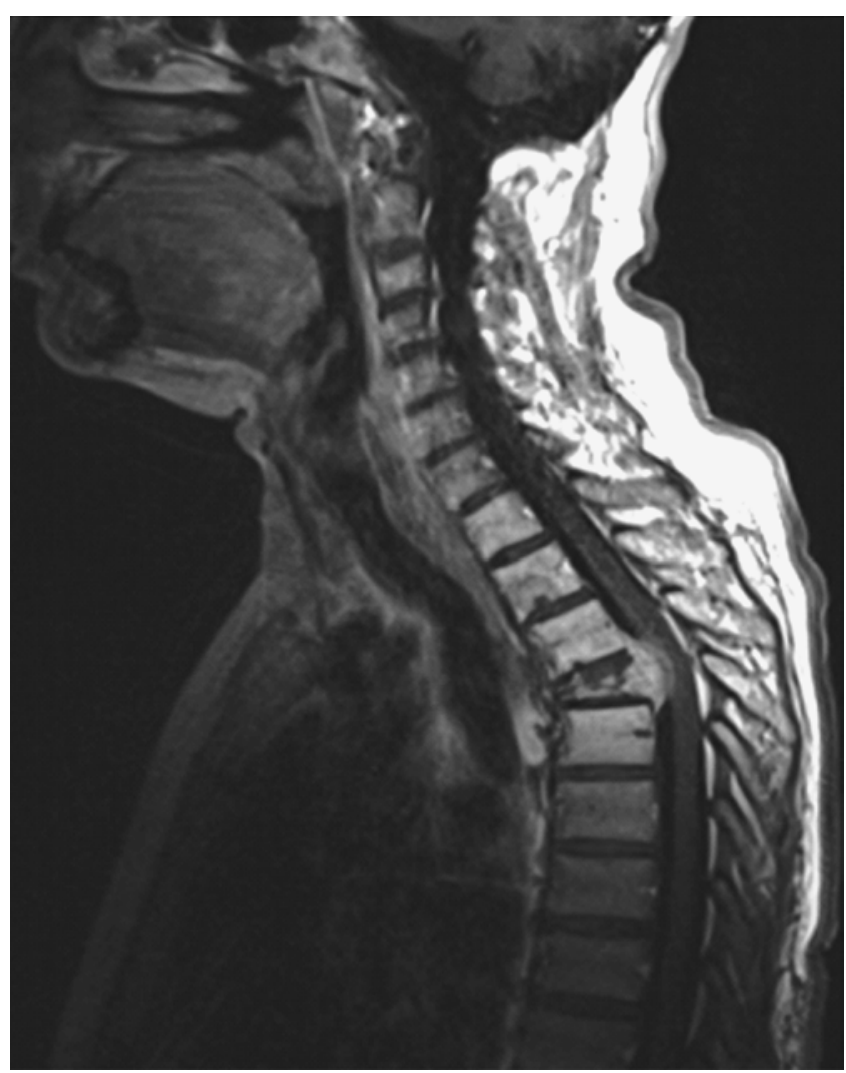

FIG. 2. Sagittal T1-weighted postcontrast MRI scan indicating a metastatic lesion to the thoracic spine with bony destruction, loss of vertebral body height, focal kyphosis, and impingement on the spinal canal.

significantly differ from that of the other patients in the current study (57.9 years) $(\mathrm{p}=0.6)$.

Eight patients underwent multiple surgical procedures. Two of these patients had additional surgery for instrumentation failure and spinal deformity or pain without local tumor recurrence. Five patients undergoing repeat surgery had local tumor recurrence in the spine requiring reoperation 1-36 months (mean 14.6 months) after the initial surgery. One patient had tumor recurrence at a distant site in the spine, and this recurrence was managed with surgical decompression and stabilization.

\section{Complications}

All adverse events that occurred within 30 days after surgery were considered early complications. Major complications were defined as those that required additional surgery, increased the length of hospital stay, or were potentially life threatening. Minor complications were defined as those that did not substantially change the clinical course of events nor increased the length of hospital stay. The perioperative complications are shown in Table 2. Major complications occurred in 9 patients (30\%) and included respiratory failure requiring reintubation, septic shock and multiple system organ failure, hospital-acquired pneumonia, rapid tumor growth leading to further neurological impairment, venous thrombosis requiring anticoagulation, pulmonary embolus, hyponatremia and delirium, and wound infection and dehiscence. Minor
TABLE 2. Perioperative complications

\begin{tabular}{cc}
\hline \multicolumn{1}{c}{ Complication } & No. of Patients (\%) \\
\hline Major & \\
\hline Respiratory failure & $2(6.7)$ \\
\hline Septic shock/MSOF & $1(3.3)$ \\
\hline Pulmonary embolus & $1(3.3)$ \\
\hline Hyponatremia/delirium & $1(3.3)$ \\
\hline Paraplegia & $1(3.3)$ \\
\hline DVT & $1(3.3)$ \\
\hline Wound infection & $2(6.7)$ \\
\hline Minor & $1(3.3)$ \\
\hline Urinary tract infection & $1(3.3)$ \\
\hline Ileus & $1(3.3)$ \\
\hline Upper-extremity DVT
\end{tabular}

DVT = deep venous thrombosis; $\mathrm{MSOF}=$ multiple system organ failure

complications were noted in 3 patients (10\%) and included urinary tract infection, ileus, and upper-extremity venous thrombosis not requiring anticoagulation.

The median length of hospital stay, not including rehabilitation, was 8.5 days (range 5-54 days). The 30-day in-hospital mortality rate was $10 \%$ (3 patients). These early deaths were attributed to septic shock and multiple system organ failure, pulmonary embolism, or respiratory failure. The mean age of the patients who died within 30 days postoperatively was 70.7 years $(p=0.09) ; 2$ of these patients had significant neurological dysfunction, with a preoperative Frankel grade of $\mathrm{C}$, and the third patient had a Frankel grade of $\mathrm{E}$ with severe back pain.

\section{Discussion}

Metastatic disease of the vertebral column is a complex clinical problem, often requiring multiple treatment modalities, including surgery, conventional radiotherapy, stereotactic radiosurgery, chemo- and immunotherapy, and pain management. Patients with RCC frequently present with metastatic spinal lesions that may cause pain and neurological dysfunction, symptoms that were present in $21(70 \%)$ and $9(30 \%)$ of our patients, respectively. Historically, the role of surgery was limited in the treatment of spinal metastases. Surgeons offered decompressive laminectomy in an attempt to salvage neurological function, whereas other patients underwent chemo- and radiotherapy. Several studies in the 1950s and 1960s actually showed that patients did worse with posterior decompressive surgery than with chemo- and radiotherapy alone. ${ }^{36}$ Therefore, surgery was largely abandoned as a primary and sole treatment for metastases of the vertebral column. Separately, checkpoint blockade, such as targeting PD-1/ PD-L1 or CTLA-4 pathways, has shown efficacy in clinical trials with acceptable safety profiles. ${ }^{17}$ In the future, targeted therapeutics and immunomodulatory therapeutics have the potential to significantly improve the clinical outcomes for patients with metastatic RCC as well as with other solid malignancies. ${ }^{17}$

The first-line treatment for metastatic RCC includes chemotherapy and radiotherapy. However, in only a few 
patients with RCC, tumors exhibit complete or total response to chemotherapy or immunotherapy.,14,15 Therefore, most patients with RCC metastases are treated with conventional radiotherapy, even though RCC is relatively radioresistant. ${ }^{9,26}$ Recent investigations into the role of stereotactic radiosurgery for RCC metastases have reported efficacy in reducing pain and providing local tumor control, but the impact of radiosurgery on patient survival is unclear. ${ }^{5,23,28}$ Furthermore, radiotherapy provides little benefit if the spinal cord is compressed by bone fragments resulting from pathological fracture. For these reasons, surgery has increasingly become a necessity in patients with RCC spinal metastases.

With the advent of new technologies for spinal fixation and vertebral column reconstruction, including pedicle screws and rods, expandable cages, anterior plating systems, and synthetic grafts, surgeons were able to offer more definitive decompression and stabilization procedures. When these newer techniques were applied, outcomes in surgical patients began to improve. Systematic reviews have reported improved outcomes over time as more surgeons employed techniques that could provide circumferential decompression and vertebral column reconstruction. ${ }^{34}$

In 2005, Patchell and colleagues reported the results from their landmark clinical trial of patients with spinal metastases managed with surgery and radiotherapy versus radiotherapy alone. ${ }^{18}$ This randomized-controlled multicenter trial sought to ascertain whether surgery was beneficial in the treatment of spinal metastases. Patients older than 18 years were eligible for inclusion in the study if they had evidence of a single area of metastatic spinal cord compression, at least 1 neurological symptom (including pain), were not completely paraplegic within 48 hours of presentation, and could receive the radiation dosage in the treatment. Patients with highly radiosensitive tumors (lymphoma, myeloma, leukemia, or germ-cell tumors) were excluded from that study. Its results indicated that surgery combined with radiotherapy is superior to radiotherapy alone. Patients who received surgery were more likely to be able to walk ( $84 \%$ vs $57 \%, \mathrm{p}=0.001$ ) and could maintain ambulation longer (122 days vs 13 days, $\mathrm{p}=0.003$ ) after treatment. The surgery plus radiotherapy patients also had significantly improved Frankel scores, American Spinal Injury Association scores, and bladder function. In addition, these patients had modestly improved survival (126 days vs 100 days, $\mathrm{p}=0.033$ ). The study by Patchell et al., which included 11 patients with RCC, helped to confirm the beneficial role of surgery in the management of spinal metastases from RCC.

In studies of patients with spinal metastases, both solitary and multiple, due to RCC, the reported length of survival has been on par with that among patients with other invasive neoplastic diseases. Sundaresan et al., studying 32 patients with metastatic RCC who were treated surgically, reported a median survival of 13 months and an improvement in pain and neurological symptoms in $78 \%$ and $70 \%$ of patients, respectively. ${ }^{26}$ Results by King et al. indicated a worse median survival of only 8 months, albeit with similar improvements (88\% and 60\%) in pain and neurological symptoms..$^{13}$ Giehl and Kluba also reported a relatively short median survival of 10 months, with $100 \%$ improvement in pain. ${ }^{7}$ Jackson et al. reported results from a cohort of 79 patients who underwent 107 operations for RCC spinal metastases. ${ }^{11}$ In this study, the median length of survival was 11.4 months, and most patients also had improvement in pain (89\% of patients) and neurological function $(65 \%)$.

More recent studies have reported modest improvement in survival among patients with RCC-derived spinal metastases. Ulmar et al. ${ }^{31}$ reported a median length of survival of 13.6 months in 37 of such patients, and Quraishi et al. ${ }^{19}$ noted a median survival of 14.1 months in 25 patients. Tatsui et al. have studied the largest patient cohort to date, consisting of 267 patients who underwent surgical treatment for RCC spinal metastases. ${ }^{27}$ These authors reported a 5-year overall survival after resection of RCC spinal metastases of $7.8 \%$, with a median length of overall survival of 11.3 months. The authors also noted that patients with a Fuhrman Grade 4 RCC, preoperative neurological deficits, or additional extraspinal metastases had a statistically significantly shorter survival than did patients without these factors. In the present study, the median survival after surgery was 11.4 months, and we found that the surgery alleviated pain and neurological dysfunction in $81 \%$ and $78 \%$ of patients, respectively. Most patients, even those with relatively advanced disease, reported less pain and preserved, if not improved, neurological function after the surgery. Similar to the findings in the study by Tatsui et al., extraspinal metastases and presence of preoperative neurological dysfunction were both significantly associated with lower survival. However, some patients with spinal metastases of RCC can have extended survival and may benefit from more aggressive surgery with the goal of minimizing local recurrence.

Radical surgical treatment, that is, total en bloc spondylectomy (TES) with margin excision, may have an especially pivotal role in selected patients with spinal metastases from RCC. Three patients in our study who underwent TES had a mean survival of 59.4 months, which likely reflected their oligometastatic presentation, a condition associated with better survival. Several authors have previously reported long-term disease-free survival in patients harboring solitary spine metastases of RCC with no other evidence of visceral or primary disease. Tomita et al. reported on the first series of patients who underwent extracapsular TES for the management of spinal metastases. ${ }^{29,30}$ Four of the patients in these studies were treated for metastatic RCC, and all 4 were alive at the time of publication (mean survival 15.5 months, range 6-32 months). Sakaura et al., studying outcomes for patients who underwent TES for solitary spinal metastases-3 of whom were treated for RCC-derived metastases-also reported excellent long-term survival among these patients (mean survival 46.7 months, range 16-60 months).$^{20}$ Sundaresan et al., reporting their results of patients treated with TES for solitary metastases (13 of whom with RCC metastases), noted that survival after TES was also encouraging (median length of survival 36 months and 5-year survival $38 \%$ ). ${ }^{25}$ In all of these studies, patients with solitary metastases of RCC had longer disease-free and overall survival than patients with many 
other common metastases. These findings highlight the potential of surgery to provide long-term disease-free survival in a select group of patients, especially those with solitary metastases of RCC.

Although radical surgical interventions can increase the likelihood of disease-free survival, the benefits of these surgeries must be weighed against the risk for increased perioperative morbidity and mortality rates, as is the case with any complex spinal surgery. Complication rates in the aforementioned surgery studies ranged from $16 \%$ to $65 \% .7,11,13,19,26,32$ The rate of major complications in the present series was $30 \%$, and these complications included respiratory failure requiring reintubation, septic shock and multiple system organ failure, hospital-acquired pneumonia, rapid tumor growth leading to further neurological compromise, venous thrombosis requiring anticoagulation, pulmonary embolus, hyponatremia and delirium, and wound infection and dehiscence. Most complications following surgery for RCC-derived spinal metastases are related to thromboembolic events or to infectious complications such as pneumonia, urinary tract infection, or wound breakdown.

Several patients in the previous studies and 2 patients in the present study underwent reintubation for respiratory failure during the postoperative period. Although many factors may contribute to respiratory failure, patients with RCC may be especially prone to developing this and other complications in the immediate postoperative period. The reason for this increased risk in these patients is that RCC metastases are usually highly vascular, resulting in high blood loss intraoperatively. The massive fluid shifts that ensue from this excessive blood loss and also from resuscitation and blood product transfusion can contribute to pulmonary edema and pneumonia, delayed mobility, skin breakdown, and other complications. Hence, many surgeons recommend preoperative arterial angiography and embolization to limit intraoperative blood loss. Preoperative spinal radiation can also predispose patients to wound complications. ${ }^{6}$ Therefore, radiotherapy should be delayed until at least 1 week after surgery if possible..$^{10}$

Factors associated with improved survival in the current study included a younger patient age, preoperative ability to ambulate, no major medical comorbidities, disease control at the primary site, and an absence of visceral and extraspinal bony metastases. In contrast, Ulmar et al. reported no significant relationship between survival and patient age or presence of extraspinal bony and visceral metastases. ${ }^{31}$ However, it stands to reason that patients with a higher burden of systemic disease, that is, those with visceral and other bony metastases, would have shorter survival. Furthermore, younger patients may have improved survival due to better overall health and possibly earlier presentation of the disease. Our findings further underscore the importance of patient selection when considering surgical intervention for spinal metastases of RCC.

There are several limitations to our current study that include the retrospective data collection and the biases inherent to studies of this design. Also, the relatively small sample size, heterogeneous patient cohort, and lack of a comparison with a medical management-only group could further limit the conclusions from this study.

\section{Conclusions}

Renal cell carcinoma is an aggressive malignant disease that frequently metastasizes to the spine. In only a few patients does the disease respond adequately to systemic chemotherapy, immunotherapy, or radiotherapy. Therefore, many patients with RCC spinal metastases undergo surgery for intractable pain or for neurological dysfunction from epidural spinal cord compression. The goals of surgery are thereby generally palliative, to decrease pain and preserve neurological function. Although the length of overall survival has not markedly changed for patients with RCC and spinal metastases, certain patients, that is, those with solitary metastases and low systemic disease burden, may benefit from aggressive surgical intervention. Longer survival may be observed in patients who are younger, are able to walk, have no major medical comorbidities, and have disease control at the primary site with no visceral or extraspinal bone metastases. However, the perioperative surgical complication rate remains high, and judicious patient selection should be pursued in an effort to minimize complications and maximize the benefits of surgery.

\section{References}

1. American Cancer Society: Cancer Facts and Figures 2013. Atlanta: American Cancer Society, 2013

2. Bell GR: Surgical treatment of spinal tumors. Clin Orthop Relat Res (335):54-63, 1997

3. Bilsky MH, Lis E, Raizer J, Lee H, Boland P: The diagnosis and treatment of metastatic spinal tumor. Oncologist 4:459469, 1999

4. Fosså SD: Interferon in metastatic renal cell carcinoma. Semin Oncol 27:187-193, 2000

5. Gerszten PC, Burton SA, Ozhasoglu C, Vogel WJ, Welch WC, Baar J, et al: Stereotactic radiosurgery for spinal metastases from renal cell carcinoma. J Neurosurg Spine 3:288295, 2005

6. Ghogawala Z, Mansfield FL, Borges LF: Spinal radiation before surgical decompression adversely affects outcomes of surgery for symptomatic metastatic spinal cord compression. Spine (Phila Pa 1976) 26:818-824, 2001

7. Giehl JP, Kluba T: Metastatic spine disease in renal cell carcinoma-indication and results of surgery. Anticancer Res 19 (2C):1619-1623, 1999

8. Harrington KD: The use of methylmethacrylate for vertebralbody replacement and anterior stabilization of pathological fracture-dislocations of the spine due to metastatic malignant disease. J Bone Joint Surg Am 63:36-46, 1981

9. Huguenin PU, Kieser S, Glanzmann C, Capaul R, Lütolf UM: Radiotherapy for metastatic carcinomas of the kidney or melanomas: an analysis using palliative end points. Int J Radiat Oncol Biol Phys 41:401-405, 1998

10. Itshayek E, Yamada J, Bilsky M, Schmidt M, Shaffrey C, Gerszten $\mathrm{P}$, et al: Timing of surgery and radiotherapy in the management of metastatic spine disease: a systematic review. Int J Oncol 36:533-544, 2010

11. Jackson RJ, Loh SC, Gokaslan ZL: Metastatic renal cell carcinoma of the spine: surgical treatment and results. J Neurosurg 94 (1 Suppl):18-24, 2001

12. Jacobsen KD, Follerås G, Fosså SD: Metastases from renal cell carcinoma to the humerus or the shoulder girdle. Br J Urol 73:124-128, 1994

13. King GJ, Kostuik JP, McBroom RJ, Richardson W: Surgical management of metastatic renal carcinoma of the spine. Spine (Phila Pa 1976) 16:265-271, 1991 
14. Margolin KA: Interleukin-2 in the treatment of renal cancer. Semin Oncol 27:194-203, 2000

15. Motzer RJ, Bander NH, Nanus DM: Renal-cell carcinoma. N Engl J Med 335:865-875, 1996

16. Nottebaert M, von Hochstetter AR, Exner GU, Schreiber A: Metastatic carcinoma of the spine. A study of 92 cases. Int Orthop 11:345-348, 1987

17. Parekh H, Rini BI: Emerging therapeutic approaches in renal cell carcinoma. Expert Rev Anticancer Ther 15:1305-1314, 2015

18. Patchell RA, Tibbs PA, Regine WF, Payne R, Saris S, Kryscio RJ, et al: Direct decompressive surgical resection in the treatment of spinal cord compression caused by metastatic cancer: a randomised trial. Lancet 366:643-648, 2005

19. Quraishi NA, Purushothamdas S, Manoharan SR, Arealis G, Lenthall R, Grevitt MP: Outcome of embolised vascular metastatic renal cell tumours causing spinal cord compression. Eur Spine J 22 (Suppl 1):S27-S32, 2013

20. Sakaura H, Hosono N, Mukai Y, Ishii T, Yonenobu K, Yoshikawa H: Outcome of total en bloc spondylectomy for solitary metastasis of the thoracolumbar spine. J Spinal Disord Tech 17:297-300, 2004

21. Schaberg J, Gainor BJ: A profile of metastatic carcinoma of the spine. Spine (Phila Pa 1976) 10:19-20, 1985

22. Smith EM, Kursh ED, Makley J, Resnick MI: Treatment of osseous metastases secondary to renal cell carcinoma. J Urol 148:784-787, 1992

23. Staehler M, Haseke N, Nuhn P, Tüllmann C, Karl A, Siebels $\mathrm{M}$, et al: Simultaneous anti-angiogenic therapy and singlefraction radiosurgery in clinically relevant metastases from renal cell carcinoma. BJU Int 108:673-678, 2011

24. Sundaresan N, Digiacinto GV, Hughes JE, Cafferty M, Vallejo A: Treatment of neoplastic spinal cord compression: results of a prospective study. Neurosurgery 29:645-650, 1991

25. Sundaresan N, Rothman A, Manhart K, Kelliher K: Surgery for solitary metastases of the spine: rationale and results of treatment. Spine (Phila Pa 1976) 27:1802-1806, 2002

26. Sundaresan N, Scher H, DiGiacinto GV, Yagoda A, Whitmore W, Choi IS: Surgical treatment of spinal cord compression in kidney cancer. J Clin Oncol 4:1851-1856, 1986

27. Tatsui CE, Suki D, Rao G, Kim SS, Salaskar A, Hatiboglu MA, et al: Factors affecting survival in 267 consecutive patients undergoing surgery for spinal metastasis from renal cell carcinoma. J Neurosurg Spine 20:108-116, 2014

28. Teh B, Bloch C, Galli-Guevara M, Doh L, Richardson S, Chiang $\mathrm{S}$, et al: The treatment of primary and metastatic renal cell carcinoma (RCC) with image-guided stereotactic body radiation therapy (SBRT). Biomed Imaging Interv J 3:e6, 2007

29. Tomita K, Kawahara N, Baba H, Tsuchiya H, Nagata S, Toribatake Y: Total en bloc spondylectomy for solitary spinal metastases. Int Orthop 18:291-298, 1994

30. Tomita K, Toribatake Y, Kawahara N, Ohnari H, Kose H: Total en bloc spondylectomy and circumspinal decompression for solitary spinal metastasis. Paraplegia 32:36-46, 1994
31. Ulmar B, Catalkaya S, Naumann U, Gerstner S, Cakir B, Schmidt R, et al: [Surgical treatment and evaluation of prognostic factors in spinal metastases of renal cell carcinoma.] $\mathbf{Z}$ Orthop Ihre Grenzgeb 144:58-67, 2006 (Ger)

32. Ulmar B, Naumann U, Catalkaya S, Muche R, Cakir B, Schmidt R, et al: Prognosis scores of Tokuhashi and Tomita for patients with spinal metastases of renal cancer. Ann Surg Oncol 14:998-1004, 2007

33. Walsh GL, Gokaslan ZL, McCutcheon IE, Mineo MT, Yasko AW, Swisher SG, et al: Anterior approaches to the thoracic spine in patients with cancer: indications and results. Ann Thorac Surg 64:1611-1618, 1997

34. Witham TF, Khavkin YA, Gallia GL, Wolinsky JP, Gokaslan ZL: Surgery insight: current management of epidural spinal cord compression from metastatic spine disease. Nat Clin Pract Neurol 2:87-94, 116, 2006

35. York JE, Walsh GL, Lang FF, Putnam JB, McCutcheon IE, Swisher SG, et al: Combined chest wall resection with vertebrectomy and spinal reconstruction for the treatment of Pancoast tumors. J Neurosurg 91 (1 Suppl):74-80, 1999

36. Young RF, Post EM, King GA: Treatment of spinal epidural metastases. Randomized prospective comparison of laminectomy and radiotherapy. J Neurosurg 53:741-748, 1980

\section{Disclosures}

Dr. Goodwin is a United Negro College Fund/Merck postdoctoral fellow and has received an award from the Burroughs Wellcome Fund. Dr. Bydon is the recipient of a research grant from DePuy Spine and serves on the clinical advisory board of MedImmune, LLC. Dr. Witham is a recipient of a research grant from Eli Lilly and Company and has received support from the Gordon and Marilyn Macklin Foundation. Dr. Gokaslan has stock ownership in US Spine and Spinal Kinetics; conducts consulting, speaking, and teaching for the AO Foundation and Research; and has support from DePuy, the Neurosurgery Research and Education Foundation, AOSpine, and AO North America. Dr. Sciubba is a consultant for Medtronic.

\section{Author Contributions}

Conception and design: Petteys, Spitz, Goodwin. Acquisition of data: Petteys, Spitz. Analysis and interpretation of data: Petteys, Spitz, Goodwin. Drafting the article: Sciubba, Petteys, Spitz, Goodwin. Critically revising the article: all authors. Reviewed submitted version of manuscript: all authors. Statistical analysis: Petteys, Spitz. Administrative/technical/material support: Sciubba, Petteys, Spitz, Goodwin, Bydon, Witham, Wolinsky, Gokaslan. Study supervision: Sciubba, Petteys, Spitz.

\section{Correspondence}

Daniel M. Sciubba, Department of Neurosurgery, Johns Hopkins University School of Medicine, 600 North Wolfe St., Meyer 7-109, Baltimore, MD 21287. email: dsciubb1@jhmi.edu. 